\title{
Article
}

\section{The effects of conventional and oval chainrings on patellofemoral loading during road cycling: an exploration using musculoskeletal simulation}

Sinclair, Jonathan Kenneth, Stainton, Philip and Sant, Benjamin

Available at http://clok.uclan.ac.uk/21138/

Sinclair, Jonathan Kenneth ORCID: 0000-0002-2231-3732, Stainton, Philip and Sant, Benjamin (2017) The effects of conventional and oval chainrings on patellofemoral loading during road cycling: an exploration using musculoskeletal simulation. Sport Sciences for Health. ISSN 1824-7490

It is advisable to refer to the publisher's version if you intend to cite from the work. http://dx.doi.org/10.1007/s11332-017-0401-6

For more information about UCLan's research in this area go to http://www.uclan.ac.uk/researchgroups/ and search for <name of research Group>.

For information about Research generally at UCLan please go to http://www.uclan.ac.uk/research/

All outputs in CLoK are protected by Intellectual Property Rights law, including Copyright law. Copyright, IPR and Moral Rights for the works on this site are retained by the individual authors and/or other copyright owners. Terms and conditions for use of this material are defined in the policies page. 

cycling: an exploration using musculoskeletal simulation. Jonathan Sinclair ${ }^{1}$, Philip Stainton ${ }^{1}$, \& Benjamin Sant ${ }^{1}$

\section{Centre for Applied Sport \& Exercise Sciences, Faculty of Health \& Wellbeing,} University of Central Lancashire, Lancashire, UK.

7 Contact Details:

8 Dr. Jonathan Sinclair

9 Centre for Applied Sport \& Exercise Sciences

10 Faculty of Health \& Wellbeing

11 Preston

12 Lancashire

PR1 2HE

e-mail: jksinclair@uclan.ac.uk

15 Keywords: Biomechanics; cycling, chainring, patellofemoral, pathology

\section{Abstract}

PURPOSE: The aim of the current investigation was to utilize a musculoskeletal simulation approach to resolve muscle forces during the pedal cycle, in order to specifically examine the effects of chainring geometry on patellofemoral loading during cycling. METHODS: Fifteen healthy male recreational cyclists rode a stationary cycle ergometer at a fixed cadence of 70 RPM in two chainring conditions (round and oval). Patellofemoral loading was explored using a musculoskeletal simulation and mathematical modelling approach. Differences between chainring conditions across the entire pedal cycle were examined using 1-dimensional statistical parametric mapping and patellofemoral force experienced per $20 \mathrm{~km}$ was explored using a paired samples t-test. 
RESULTS: No significant $(\mathrm{P}>0.05)$ differences in patellofemoral force or stress were found

27 throughout the pedal cycle between chainring conditions. It was also shown that no significant $(\mathrm{P}>0.05)$ differences in patellofemoral force per $20 \mathrm{~km}$ joint were evident (round $38576.40 \mathrm{~N} / \mathrm{kg} \cdot \mathrm{s} \&$ oval $=35637.00 \mathrm{~N} / \mathrm{kg} \cdot \mathrm{s})$.

CONCLUSIONS: The current analysis found no effects of chainring geometry, on the forces experienced by the patellofemoral joint during the pedal cycle.

\section{Introduction}

During linear road cycling using traditional circular chainrings, the application of tangential force is lowest when the crank in the vertically aligned, either at 0 or $180^{\circ}$ of the pedal cycle, and maximal when the crank is horizontally aligned (1). The points during the pedal cycle where tangential force is lowest are typically referred to as upper and lower dead points (2). In an attempt to improve road cycling performance and maximize the application of effective force during the pedal cycle, oval chainrings were introduced, whereby the axes of the chainring are not perpendicular (3). This shape means that the moment arm of the force being applied to the chain is reduced at the dead points of the pedal cycle but increased when the crank is horizontally aligned (3). This optimizes the period of the pedal cycle in which tangential force is produced, and correspondingly reduces the time spent in the upper and lower dead points (4).

Quantitative analyses investing performance parameters with oval chainrings have shown inconsistent findings. Hintzy et al., (4) showed that peak power output was significantly higher when using a non-circular chainrings during short duration maximal spring cycling. Hintzy \& Horvais, (5) similarly found that higher maximal aerobic power was attained when using a non-circular chainring during maximal incremental tests. Horvais et al., (6) examined 
mechanical and physiological parameters during 8 minute submaximal and $8 \mathrm{~s}$ maximal tests.

During the submaximal test the oval chainring produced lower crank torques at $0^{\circ}$ and $180^{\circ}$ and greater torques at $90^{\circ}$ of the pedal cycle. During the sprint test, the biceps femoris exhibited a longer burst of activation in the oval chainring condition. Conversely, Cordova et al., (2) showed that there were no significant differences in physiological responses during an incremental test until exhaustion. Similarly, Peiffer \& Abbiss, (7) found that there were no differences in physiological and performance parameters between oval and round chainrings during a $10 \mathrm{~km}$ cycling time trial. Finally, Dagnese et al., (8) similarly showed that there were no significant differences in lower extremity muscle activation magnitude between oval and round chainrings.

Further to this, Bisi et al., (9) showed that oval chainrings altered lower extremity joint kinetics, with reductions of $6 \%$ in the knee joint moment, which they identified may have implications for chronic injury prevention at this joint. Importantly the knee joint is the musculoskeletal structure most susceptible to chronic pathology in cyclists (10). Specifically, patellofemoral pain is the most frequently experienced condition, affecting $36 \%$ of all regular cyclists' and accounting for more than $57 \%$ of all time-loss injuries (11). Despite the incidence of patellofemoral pain in cyclists it has received a paucity of attention in scientific literature in relation to other athletic disciplines. Therefore, further exploration of this condition is clearly warranted in cycling specific analyses.

Patellofemoral pain is initiated by activities that place frequent and excessive mechanical loads at the joint $(12,13)$. Therefore, quantification of patellofemoral loading is important in cycling specific activities as we seek to understand more about this condition and the potential mechanisms that may be important to prevent the high incidence of patellofemoral 
pain. Although validated mathematical models of the patellofemoral joint are available in biomechanical literature $(14,15)$, they typically require inverse joint dynamics to resolve muscle kinetics as input parameters into the musculoskeletal algorithm. Whilst this is suitable for movements which involve full foot contact with a force platform, this is not available for cycling specific analyses, which may help to explain the lack of scientific attention concerning to patellofemoral pain in road cycling.

However, advances in musculoskeletal modelling have led to the development of bespoke software which allows skeletal muscle force distributions to be simulated during movement using motion capture based data (16). To date, such approaches have not yet been utilized in cycling specific analyses. The aim of the current investigation was therefore to utilize a musculoskeletal simulation approach to resolve muscle forces during cycling to examine the effects of chainring geometry on patellofemoral loading during the pedal cycle. A study of this nature may provide important clinical information regarding the effects of different chainring technology on the susceptibility of road cyclists to patellofemoral pain.

\section{Materials \& methods}

\section{Participants}

Fifteen male recreational cyclists, who habitually utilized round chainrings for their training volunteered to take part in this study. Cyclists were required to have at least 2 years of road cycling experience and be free from musculoskeletal pathology at the time of data collection.

The mean characteristics of the participants were; age $28.11 \pm 5.11$ years, height $1.80 \pm 0.10$ $\mathrm{m}$ and body mass $75.10 \pm 8.22 \mathrm{~kg}$. The procedure utilized for this investigation was approved by the University of Central Lancashire, Science, Technology, Engineering and Mathematics, ethical committee (Ref: 511) and all participants provided written informed consent 
Procedure

Participants rode a stationary cycle ergometer SRM 'Indoor Trainer' (SRM, Schoberer, 104 Germany) for 10 minutes at a fixed cadence of 70 RPM using a 52x15 gear ratio. To ensure that the current investigation examined only the effects of the different chainrings, the set-up parameters were constructed in accordance with previous recommendations (17), and standardized between the two conditions. Cycling shoes (Northwave Sonic 2 Plus Road Shoes, Northwave, Italy), pedals (Look Keo Classic 2, Look, Cedex, France) and cleats (Look Keo Grip, $4.5^{\circ}$ float, Look, Cedex, France) were consistent across all trials, and adjusted so that the 1st metatarsal head was positioned superior to the pedal spindle (18). The participants were provided with continuous visual feedback regarding their cadence, which was visible via the SRM head unit (Powercontrol V, SRM, Schoberer, Germany).

The participants rode in two conditions one with a traditional round chainring (SRM power, SRM, Schoberer, Germany) and one using an oval shaped chainring (Osymetric, standard, USA), with a crank length of $172.5 \mathrm{~mm}$. To prevent any order effects in the experimental data, the order in which participants rode in each chainring condition was counterbalanced and a standardized rest period of 10 minutes was allowed between trials. The ergometer setup was organized based on each participant own preference and maintained between the two chainring conditions.

Kinematic information from the lower extremity joints was obtained using an eight camera motion capture system (Qualisys Medical AB, Goteburg, Sweden) using a capture frequency of $250 \mathrm{~Hz}$. To define the anatomical frames of the thorax, pelvis, thighs, shanks and feet retroreflective markers were placed at the C7, T12 and xiphoid process landmarks and also 
positioned bilaterally onto the acromion process, iliac crest, anterior superior iliac spine

127 (ASIS), posterior super iliac spine (PSIS), medial and lateral malleoli, medial and lateral femoral epicondyles, greater trochanter, calcaneus, first metatarsal and fifth metatarsal. Carbon-fibre tracking clusters comprising of four non-linear retroreflective markers were positioned onto the thigh and shank segments. In addition to these the foot segments were tracked via the calcaneus, first metatarsal and fifth metatarsal, the pelvic segment was tracked using the PSIS and ASIS markers and the thorax segment was tracked using the T12, C7 and xiphoid markers. Static calibration trials were obtained with the participant in the anatomical position in order for the positions of the anatomical markers to be referenced in relation to the tracking clusters/markers. A static trial was conducted with the participant in the anatomical position in order for the anatomical positions to be referenced in relation to the tracking markers, following which those not required for dynamic data were removed.

\section{Processing}

Dynamic trials were digitized using Qualisys Track Manager in order to identify anatomical and tracking markers then exported as C3D files to Visual 3D (C-Motion, Germantown, MD, USA). Marker data were smoothed using a cut-off frequency $12 \mathrm{~Hz}$ using a low-pass Butterworth 4th order zero-lag filter; this was established using residual analysis similar to Sinclair et al., (19).

Data from five pedal cycles in each chainring condition were exported from Visual 3D into OpenSim 3.3 software (Simtk.org). The five extracted pedal cycles were obtained during minutes 4-6 of the experimental protocol, and the pedal cycle itself was delineated in accordance with Sinclair et al., (19). A validated musculoskeletal model (gait2392) with 8 segments, 19 degrees of freedom and 92 musculotendon actuators (Delp et al., 2007) was 
used to resolve muscle kinetics during the pedal cycle. The model was scaled for each participant using the anthropometrics and segment inertial properties generated from the static trial to account for the dimensions of each athlete. We firstly performed a residual reduction algorithm (RRA) within OpenSim, this utilizes the inverse kinematics that were exported from Visual 3D. The RRA calculates the joint torques required to re-create the dynamic motion. The RRA calculations produced route mean squared errors $<2^{\circ}$, which correspond with the recommendations for good quality data. Following the RRA, the computed muscle control (CMC) procedure was then employed to estimate a set of muscle force patterns allowing the model to replicate the required kinematics (20). The CMC procedure works by estimating the required muscle forces to produce the net joint torques.

Patellofemoral loading during cycling was quantified using a model adapted from van Eijden et al., (14) in accordance with the protocol of Willson et al., (21). A key drawback of this model is that co-contraction of the knee flexor musculature is not accounted for. Taking this into account, summed hamstring and gastrocnemius forces derived from the CMC procedure were multiplied by their estimated knee joint muscle moment arms as a function of knee flexion angle (22) and then added together to determine the knee flexor torque during the pedal cycle. In addition to this the knee extensor torque was also calculated by dividing the summed quadriceps forces by this muscle groups' knee joint muscle moment arms as a function of knee flexion angle (14). The knee flexor and extensor torques were then summed and subsequently divided by the quadriceps muscle moment arm (14) to obtain quadriceps force adjusted for co-contraction of the knee flexor muscles (21). Patellofemoral force was quantified by multiplying the derived quadriceps force by a constant which was obtained by using the data of Eijden et al., (14). Finally, patellofemoral joint stress was quantified by dividing the patellofemoral force by the patellofemoral contact area. Patellofemoral contact 
areas were obtained by fitting a polynomial curve to the sex specific data of Besier et al.,

177 (12), who estimated patellofemoral contact areas as a function of the knee flexion angle using MRI.

Following this the patellofemoral force, muscle force and knee flexion angle data for each participant during the entire pedal cycle were extracted and time normalized to 101 data points. All joint and muscle force parameters were subsequently normalized by dividing the net values by body mass $(\mathrm{N} / \mathrm{kg})$. In addition to this, the patellofemoral force integral during the pedal cycle was obtained using a trapezoidal function. As cycling requires a uniquely recurrent movement pattern, with a significant number of pedal cycles to complete typical training/ competitive distances, the total patellofemoral force experienced per $20 \mathrm{~km}$ was also extracted. This was resolved firstly by quantifying the velocity of the bicycle using the gear ratio, cadence and typical wheel diameter/ tire width. Using this information (neglecting for air resistance and assuming that the velocity was uniform) the time taken to cycle $20 \mathrm{~km}$ could then be calculated. From this the number of pedal cycles required to complete the aforementioned distance was calculated. Finally, in accordance with Sinclair et al., (23) the patellofemoral force integral was multiplied by the number of pedal cycles necessary to cycle $20 \mathrm{~km}$ to extract the patellofemoral force experienced during this distance.

Analyses

Differences in patellofemoral and muscle forces across the entire pedal cycle were examined using 1-dimensional statistical parametric mapping with MATLAB 2017a (MATLAB, MathWorks, Natick, USA), in accordance with (24), using the source code available at http://www.spm1d.org/. For patellofemoral force per $20 \mathrm{~km}$, descriptive statistics of means, standard deviations (SD) and $95 \%$ confidence intervals $(95 \% C I)$ were calculated for both 
chainring conditions. Differences in patellofemoral force per $20 \mathrm{~km}$ between chainring

202

203

204 205

206

207

208

209

210

211

212

213

214

215

216

217

218

219

220

221

222

223

224

5
conditions were examined using a paired samples t-test. Effect sizes were calculated using partial eta ${ }^{2}\left(\mathrm{p \eta}^{2}\right)$. The alpha $(\alpha)$ level for statistical significance was set at the 0.05 level throughout. Discrete statistical tests were conducted using SPSS v23.0 (SPSS, USA).

\section{Results}

Table 1 and figures 1-6 present differences in muscle kinetics and patellofemoral loading as a function of the different chainring conditions.

\section{Patellofemoral loading}

No significant differences $(\mathrm{P}>0.05)$ in patellofemoral loading were evident across the pedal cycle as a function of the different chainring conditions (Figure 1-2). In addition, no significant $(\mathrm{P}>0.05)$ differences in patellofemoral force per $20 \mathrm{~km}$ were evident between chainring conditions (Table 1).

@@@ TABLE1 NEAR HERE @ @
$@ @ @$ FIGURE 1 NEAR HERE @ @
$@ @ @$ FIGURE 2 NEAR HERE @ @

\section{Muscle kinetics}

No significant differences $(\mathrm{P}>0.05)$ in muscle kinetics were evident across the pedal cycle as a function of the different chainring conditions (Figure 3-6). 


\section{Discussion}

The aim of the current investigation was to examine the effects of chainring geometry on patellofemoral loading throughout the pedal cycle using a statistical parametric mapping approach. To the authors knowledge this represents the first investigation to quantify the effects of different chainrings on the loads experienced by this joint throughout the pedal cycle. Given the high incidence of patellofemoral pain in road cyclists this investigation may provide important information concerning the effects of different bicycle technology regarding cyclists' susceptibility to chronic pathologies.

The key observation from the current study is that no significant differences in patellofemoral loading parameters were observed at any point during the pedal cycle as a function of the different chainring geometries examined as a part of this investigation. This opposes the proposition initiated by Bisi et al., (9) which denoted that the reduction in knee joint moment observed in the oval chainring condition may have implications for chronic injury prevention at this joint. This disagreement is likely due to the distinction between joint inverse dynamics and specific indices of joint loading; it has been shown that alterations in joint torque do not necessarily reflect changes in joint loading (25). Therefore, it can be concluded from this investigation that chainring geometry does not appear to influence patellofemoral loading during the pedal cycle.

It is proposed that this finding relates to the lack of statistical differences in muscle kinetics between the two conditions. No differences in knee flexor/ extensor muscle kinetics were 
observed between round and oval chainrings at any point during the pedal cycle. Importantly,

252 Herzog et al., (26) have shown that muscles are the main determinant of joint forces. In addition, the current study showed that there were no differences in knee joint kinematics throughout the pedal cycle, between the two chainring conditions. Taking into account that patellofemoral contact area (12) and knee flexor/ extensor muscle moment arms $(14,22)$, were modelled as a function of the knee joint angle, provides further insight into the absence of statistical differences in patellofemoral loading between conditions.

There is a clear link between excessive patellofemoral joint kinetics and the aetiology and progression of patellofemoral pain $(12,13)$. The current study represents the first investigation firstly to explore patellofemoral kinetics during the pedal cycle using a mathematical model that accounts for co-contraction of the knee flexor musculature but also to quantify the loads experienced by this joint during a typical cycling training/ competitive distance. The findings show that cyclists experience considerable patellofemoral loads, indeed although the peak forces during the pedal cycle (round $=27.86 \&$ oval $=25.92 \mathrm{~N} / \mathrm{kg}$ ) are lower than those during the stance phase of running which range between; 31.29 - 76.4 $\mathrm{N} / \mathrm{kg}$ (27-29); the cumulative loads observed during the current study (round $=38576.40$ $\mathrm{N} / \mathrm{kg} \cdot \mathrm{s} \&$ oval $=35637.00 \mathrm{~N} / \mathrm{kg} \cdot \mathrm{s}$ ) over the same linear distance are larger than those experienced during running which range between; $27774.07-30721.33 \mathrm{~N} / \mathrm{kg} \cdot \mathrm{s}(23)$. This is a thought-provoking statistic which helps to contextualize the high incidence of patellofemoral pain in cyclists and highlights the lack of scientific research into the patellofemoral joint in cycling. There is currently a clear requirement for both prophylactic and treatment intervention studies in cycling which are almost entirely absent in scientific literature. This will serve to address the underlying epidemiological factors associated with patellofemoral 
pain in cyclists and most importantly initiate a body of clinical research concerning sustained conservative treatment modalities.

\section{Limitations \& conclusions}

A limitation of the current investigation is that only healthy cyclists were examined. It is currently unknown whether cyclists with patellofemoral pain differ in their joint loading in comparison to healthy athletes, but Dieter et al., (10) demonstrated that cyclists with patellofemoral pain exhibit altered muscle activation patterns compared to healthy controls. Therefore generalizations of the current observations results to cyclists with existing patellofemoral symptoms should be made with caution. A second potential drawback is that patellofemoral loading was extracted using a mathematical modelling approach. Whist this procedure was considered an improvement over previous approaches in that co-contraction of the knee flexor musculature was accounted for; individualized muscle moment arms and patellofemoral contact areas are still not available within biomechanical literature. Finally, that the current investigation examined cyclists who do not habitually ride using oval shaped chainrings, may limit the generalizability of the results, which may have differed had the riders been more familiar with this chainring condition. Therefore, it is important for the current investigation to be repeated using cyclists who habitually utilize oval chainrings, which will allow more definitive conclusions to be drawn.

In conclusion, although the effects of altering the geometry of the chainring have been investigated previously, current knowledge regarding the effects of oval chainrings on patellofemoral loading during cycling is lacking. This study consequently adds to the current literature base in the field of biomechanics by presenting a comprehensive examination of patellofemoral loading parameters during linear cycling with both round and oval chainrings. 
300

301

302

303

304

305

306

307

308

309

310

311

312

313

314

315

316

317

318

319

320

321

322

The findings from current work show that the no differences in patellofemoral loading were evident between the two chainring conditions. This therefore indicates that chainring geometry does not significantly influence patellofemoral loading linked to the aetiology of patellofemoral pain during cycling.

\section{Acknowledgements}

We thank Gareth Shadwell for his technical assistance.

We thank Todd Pataky, Mark Robinson and Jos Vanrenterghem for their website (http://www.spm1d.org/) and for generously providing the source code for this experiment.

\section{Compliance with ethical standards}

\section{Conflict of interest}

We declare that we have no conflict of interest.

\section{Ethical approval}

All procedures performed in studies involving human participants were in accordance with the ethical standards of the institutional and the declaration of Helsinki.

\section{Informed consent}

All of the subjects provided written consent.

\section{References}

1. Ericson MO, Nisell R (1988). Efficiency of pedal forces during ergometer cycling. Int J Sports Med 9: 118-122. DOI:10.1055/s-2007-1024991 
2. Cordova A, Latasa I, Seco J, Villa G, Rodriguez-Falces J (2014). Physiological responses during cycling with oval chainrings (Q-Ring) and circular chainrings. J Sports Sci Med 13: 410-416.

3. Strutzenberger G, Wunsch T, Kroell J, Dastl J, Schwameder H (2014). Effect of chainring ovality on joint power during cycling at different workloads and cadences. Sports Biomech 13: 97-108. http://dx.doi.org/10.1080/14763141.2014.908946

4. Hintzy F, Grappe F, Belli A (2016). Effects of a Non-Circular Chainring on Sprint Performance During a Cycle Ergometer Test. J Sports Sci Med 15: 223-228.

5. Hintzy F, Horvais N (2016). Non-circular chainring improves aerobic cycling performance in non-cyclists. Eur J Sport Sci 16: 427-432. http://dx.doi.org/10.1080/17461391.2015.1086817

6. Horvais N, Samozino P, Zameziati K, Hautier C, Hintzy F (2007). Effects of a noncircular chainring on muscular, mechanical and physiological parameters during cycle ergometer tests. Isokinet Exerc Sci 15: 271-279.

7. Peiffer JJ, Abbiss CR (2010). The influence of elliptical chainrings on $10 \mathrm{~km}$ cycling time trial performance. Int $\mathrm{J}$ Sports Physiol Perform 5: 459-468. https://doi.org/10.1123/ijspp.5.4.459

8. Dagnese F, Carpes FP, Martins ED, Stefanyshyn D, Mota CB (2011). Effects of a noncircular chainring system on muscle activation during cycling. J Electromyogr Kinesiol 21: 13-17. DOI: 10.1016/j.jelekin.2010.02.005

9. Bisi MC, Stagni R, Gnudi G, Cappello A (2010). Non-circular chain ring allows a reduction of joint loading in cycling. J Mech Med Biol 10: 113-122. DOI: http://dx.doi.org/10.1142/S0219519410003228 
10. Dieter BP, McGowan CP, Stoll SK, Vella CA (2014). Muscle activation patterns and patellofemoral pain in cyclists. Med Sci Sports Exerc 46: 753-761. DOI: 10.1249/MSS.0000000000000153

11. Clarsen B, Krosshaug T, Bahr R (2010). Overuse injuries in professional road cyclists. Am J Sports Med 38: 2494-2501. DOI: 10.1177/0363546510376816

12. Besier TF, Draper CE, Gold GE, Beaupre GS, Delp SL. Patellofemoral joint contact area increases with knee flexion and weight-bearing. J Orthop Res 2005: 23: 345-350. DOI: $10.1016 /$ j.orthres.2004.08.003

13. Ho KY, Blanchette MG, Powers CM (2012). The influence of heel height on patellofemoral joint kinetics during walking. Gait Posture 36: 271-275. DOI: 10.1016/j.gaitpost.2012.03.008

14. van Eijden TM, Kouwenhoven E, Verburg J, Weijs WA (1986). A mathematical model of the patellofemoral joint. J Biomech 19: 219-229. https://doi.org/10.1016/0021-9290(86)90154-5

15. Elias JJ, Wilson DR, Adamson R, Cosgarea AJ (2004). Evaluation of a computational model used to predict the patellofemoral contact pressure distribution. J Biomech 37: 295-302. https://doi.org/10.1016/S0021-9290(03)00306-3

16. Delp SL, Anderson FC, Arnold AS, Loan P, Habib A, John CT, Thelen DG (2007). OpenSim: open-source software to create and analyze dynamic simulations of movement. IEEE Trans Biomed Eng 54: 1940-1950. DOI: 10.1109/TBME.2007.901024

17. de Vey Mestdagh, K. (1998). Personal perspective in search of an optimum cycling posture. Appl Ergon 29: 325-334. DOI:10.1016/S0003-6870(97)00080-X 
18. Menard, M., Domalain, M., Decatoire, A., \& Lacouture, P. (2016). Influence of saddle setback on pedalling technique effectiveness in cycling. Sport Biomech 15: 462-472. DOI: http://dx.doi.org/10.1080/14763141.2016.1176244

19. Sinclair J, Hebron J, Atkins S, Hurst H, Taylor PJ. (2014). The influence of 3D kinematic and electromyographical parameters on cycling economy. Acta Bioeng Biomech 16: 91-97. DOI: 10.5277/ABB-00049-2014-02

20. Thelen DG, Anderson FC, Delp SL (2003). Generating dynamic simulations of movement using computed muscle control. J Biomech 36: 321-328. DOI:10.1016/S0021-9290(02)00432-3

21. Willson JD, Ratcliff OM, Meardon SA, Willy RW (2015). Influence of step length and landing pattern on patellofemoral joint kinetics during running. Scand J Med Sci Sports 25: 736-743. DOI: 10.1111/sms. 12383

22. Spoor CW, van Leeuwen JL (1992). Knee muscle moment arms from MRI and from tendon travel. J Biomech 25: 201-206. https://doi.org/10.1016/0021-9290(92)90276-7

23. Sinclair J, Richards J, Selfe J, Fau-Goodwin J, Shore H. (2016). The influence of minimalist and maximalist footwear on patellofemoral kinetics during running. J App Biomech 32: 359-364. DOI:10.1123/jab.2015-0249

24. Pataky TC, Robinson MA, Vanrenterghem J (2016). Region-of-interest analyses of one-dimensional biomechanical trajectories: bridging OD and 1D theory, augmenting statistical power. Peer J 4: 2652-2664. DOI: 10.7717/peerj.2652

25. Kutzner I, Trepczynski A, Heller MO, Bergmann G (2013). Knee adduction moment and medial contact force-facts about their correlation during gait. PLoS One 8: e81036. $\quad$ https://doi.org/10.1371/journal.pone.0081036 
404

405

406

407

408

409

410

411

412

413

414

415

26. Herzog W, Longino D, Clark A (2003). The role of muscles in joint adaptation and degeneration. Langenbecks Arch Surg 388: 305-315. DOI:10.1007/s00423-003-04026

27. Kulmala JP, Avela J, Pasanen K, Parkkari J (2013). Forefoot strikers exhibit lower running-induced knee loading than rearfoot strikers. Med Sci Sports Exerc 45: 23062313. DOI: 10.1249/MSS.0b013e31829efcf7

28. Bonacci J, Vicenzino B, Spratford W, Collins P (2014). Take your shoes off to reduce patellofemoral joint stress during running. $\mathrm{Br} \mathrm{J}$ Sports Med 48: 425-8. DOI:10.1136/bjsports-2013-092160.

29. Sinclair J (2014). Effects of barefoot and barefoot inspired footwear on knee and ankle loading during running. Clin Biomech 29: 395-399. DOI: 10.1016/j.clinbiomech.2014.02.004

\section{Figure labels}

Figure 1: Patellofemoral force (a.), stress (b.) and (c.) sagittal plane knee angle as a function of chainring geometry.

Figure 2: Comparison of patellofemoral force (a.), stress (b.) and (c.) sagittal plane knee angle between conditions, positive values indicate that the round chainring values exceed those in the oval condition (SPM (t) denotes the $t$ value and critical thresholds for statistical significance are denoted via the horizontal dotted lines).

Figure 3: Knee extensor kinetics (a.), rectus femoris (b.), vastus lateralis (c.) and vastus medialis (d.) vastus intermedius as a function of chainring geometry.

Figure 4: Comparison of rectus femoris (a.), vastus lateralis (b.), vastus medialis (c.) and (d.) vastus intermedius between conditions, positive values indicate that the round chainring 
416 values exceed those in the oval condition (SPM (t) denotes the $t$ value and critical thresholds 417 for statistical significance are denoted via the horizontal dotted lines).

418 Figure 5: Knee flexor kinetics (a.) semimembranosus, (b.) semitendinosus, (c.) biceps femoris 419 short head, (d.) biceps femoris long head, (e.) lateral gastrocnemius and (f.) medial 420 gastrocnemius as a function of chainring geometry.

421 Figure 6: Comparison of semimembranosus (a.), semitendinosus (b.), biceps femoris short 422 head (c.), biceps femoris long head (d.), (e.) lateral gastrocnemius and (f.) medial 423 gastrocnemius positive values indicate that the round chainring values exceed those in the 424 oval condition (SPM (t) denotes the $t$ value and critical thresholds for statistical significance 425 are denoted via the horizontal dotted lines). 
Tables

Table 1: Patellofemoral force per $20 \mathrm{~km}$ (Mean, SD \& 95\% Cl) as a function of chainring geometry.

\begin{tabular}{|c|c|c|c|c|c|c|c|c|}
\hline & \multicolumn{3}{|c|}{ Round } & \multicolumn{3}{|c|}{ Oval } & \\
\hline & Mean & SD & $95 \% \mathrm{Cl}$ & Mean & SD & $95 \% \mathrm{Cl}$ & P-value & $\mathrm{pq}^{2}$ \\
\hline Patellofemoral force per $20 \mathrm{~km}(\mathrm{~N} / \mathrm{kg} \cdot \mathrm{s})$ & 38576.40 & 10796.83 & $31716.42-45436.38$ & 35637.00 & 8306.64 & 30359.21-40914.78 & 0.52 & 0.04 \\
\hline
\end{tabular}

